\title{
The Crank-Nicolson finite element method for the 2D uniform transmission line equation
}

\author{
Hulin Ren ${ }^{1}$, Yiting Fan ${ }^{1}$ and Zhendong Luo ${ }^{2 *}$
}

"Correspondence:

zhdluo@ncepu.edu.cn

${ }^{2}$ School of Mathematics and Physics, North China Electric Power University, Beijing, China Full list of author information is available at the end of the article

\begin{abstract}
We develop the Crank-Nicolson finite element (CNFE) method for the two-dimensional (2D) uniform transmission line equation, study the stability and existence as well as error estimates for the CNFE solutions of the 2D uniform transmission line equation by strict theoretical approaches. We verify the correctness of the obtained theoretical results by means of numerical tests.

MSC: $65 \mathrm{~N} 12 ; 65 \mathrm{~N} 30 ; 65 \mathrm{M} 15$

Keywords: Crank-Nicolson finite element method; Uniform transmission line equation; Stability and existence; Error estimate; Numerical test
\end{abstract}

\section{Introduction}

Let $\Theta$ be an open bounded region in $\mathbb{R}^{2}$. We consider the uniform transmission line equation in the region $\Theta$ :

$$
\begin{cases}\sigma V_{t}+V_{t t}-\Delta V+\delta V=F(x, y, t), & (x, y) \in \Theta, t \in(0, T), \\ V(x, y, t)=V_{0}(x, y, t), & (x, y) \in \partial \Theta, t \in(0, T), \\ V(x, y, 0)=H_{0}(x, y), \quad V_{t}(x, y, 0)=H_{1}(x, y), & (x, y) \in \Theta,\end{cases}
$$

where $V$ represents the unknown voltage or current, $V_{t}=\partial V / \partial t, V_{t t}=\partial^{2} V / \partial t^{2}, \Delta V=$ $\partial^{2} V / \partial x^{2}+\partial^{2} V / \partial y^{2}, F(x, y, t)$ represents the source term, $V_{0}(x, y, t)$ is the boundary value, $H_{0}(x, y)$ and $H_{1}(x, y)$ stand for initial values, $T$ is the final moment, and $\sigma=R G(\hat{C} L)^{-1}>0$ and $\delta=(\hat{C} R+L G)(\hat{C} L)^{-1}>0$ due to $G$ standing for the conductivity in the uniform transmission line, $R$ for the impedance in the uniform transmission line, $L$ for the inductance, and $\hat{C}$ for the electric capacity in the uniform transmission line. For convenience, we will premise that $V_{0}(x, y, t)=0$ in theoretical discussion.

The uniform transmission line equation known as the telegraph equation is a crucial physical equation and has largely extensive applications (see $[1,2])$. Nevertheless, when its source term, boundary value, or initial values are complex, or its coefficients $\sigma$ and $\delta$ are discontinuous, it has no genuine solution, so that we have to rely on numeric solutions.

The finite element (FE) method is one of most effective numerical methods and is used to solve many partial differential equations (see, e.g., [3-6]), whereas the Crank-Nicolson FE

(c) The Author(s) 2020. This article is licensed under a Creative Commons Attribution 4.0 International License, which permits use, sharing, adaptation, distribution and reproduction in any medium or format, as long as you give appropriate credit to the original author(s) and the source, provide a link to the Creative Commons licence, and indicate if changes were made. The images or other third party material in this article are included in the article's Creative Commons licence, unless indicated otherwise in a credit line to the material. If material is not included in the article's Creative Commons licence and your intended use is not permitted by statutory regulation or exceeds the permitted use, you will need to obtain permission directly from the copyright holder. To view a copy of this licence, visit http://creativecommons.org/licenses/by/4.0/. 
(CNFE) method has not been used to solve the uniform transmission line equation. Hence, in this paper, we intend to develop the CNFE method for the 2D uniform transmission line equation, analyze the stability and existence as well as errors for the CNFE solutions of the $2 \mathrm{D}$ uniform transmission line equation, and verify the correctness of the obtained theoretical results via numerical tests.

Although the collocation spectral method, space-time finite element method, and finite difference scheme (see [7-14]) have been used to solve the uniform transmission line equation, they are different from the CNFE method, whereas the CNFE method has more merits than the methods mentioned; for example, the CNFE method for the 2D uniform transmission line equation is unconditionally stable, resulting in that it can ensure that the numerical solution is absolutely convergent and can obtain optimal order error estimates for the CNFE solutions unmatched for the above methods.

The paper is organized as follows. In Sect. 2, we firstly construct the CNFE model for the $2 \mathrm{D}$ uniform transmission line equation and analyze the existence, uniqueness, stability, and convergence of the CNFE solutions. Afterward, in Sect. 3, we use some numerical tests to verify the correctness of the obtained theoretical results. Lastly, we summarize the main conclusions and give some prospection in Sect. 4.

\section{The CNFE method for the 2D uniform transmission line equation}

\subsection{The weak form of the 2D uniform transmission line equation}

We will use the classical Sobolev spaces and norms (see, e.g., $[15,16]$ ). Let $\mathbb{U}=H_{0}^{1}(\Theta)$. Using Green's formula, we can derive the following weak form for the 2D uniform transmission line equation (1).

Problem 1 Find $V \in H^{2}(0, T ; \mathbb{U})$ such that

$$
\left\{\begin{array}{l}
\left(V_{t t}, \vartheta\right)+(\nabla V, \nabla \vartheta)+\sigma\left(V_{t}, \vartheta\right)+\delta(V, \vartheta)=(F, \vartheta), \quad \vartheta \in \mathbb{U} \\
V(x, y, 0)=H_{0}(x, y), \quad V_{t}(x, y, 0)=H_{1}(x, y), \quad(x, y) \in \Theta
\end{array}\right.
$$

where $(\cdot, \cdot)$ stands for the inner product in $L^{2}(\Theta)$, and $a(\omega, v)=(\nabla \omega, \nabla v)$.

For Problem 1, we have the following conclusion of the existence, uniqueness, and stability of the weak solution.

Theorem 1 If $F \in L^{2}\left(0, T ; L^{2}(\Theta)\right), H_{1} \in L^{2}(\Theta)$, and $H_{0} \in H^{1}(\Theta)$, then the weak formulation (2) has a unique weak solution $V \in H^{2}(0, T ; \mathbb{U})$ meeting the stability

$$
\left\|V_{t}\right\|_{0}+\|V\|_{1} \leq \tilde{C}\left(\left\|H_{0}\right\|_{1}+\left\|H_{1}\right\|_{0}+\|F\|_{L^{2}\left(H^{-1}\right)}\right),
$$

where $\tilde{C}=2 \sqrt{\max \{1, \delta, 1 /(2 \sigma)\} / \min \{1, \delta\}}$.

Proof Since (2) is a linear equation system with respect to unknown function $V$, it has a unique solution if and only if it has merely a zero solution as $F(x, y, t)=H_{0}(x, y)=$ $H_{1}(x, y)=0$.

Taking $\vartheta=V_{t}$ in the first equation in Problem 1 , we get

$$
\frac{\mathrm{d}\left\|V_{t}\right\|_{0}^{2}}{2 \mathrm{~d} t}+\frac{\mathrm{d}\|\nabla V\|_{0}^{2}}{2 \mathrm{~d} t}+\sigma\left\|V_{t}\right\|_{0}^{2}+\delta \frac{\mathrm{d}\|V\|_{0}^{2}}{2 \mathrm{~d} t}=\left(F, V_{t}\right)
$$


Integrating (4) from 0 to $t \in[0, T]$ and using the Hölder and Cauchy inequalities, we get

$$
\begin{aligned}
& \left\|V_{t}\right\|_{0}^{2}+\|\nabla V\|_{0}^{2}+2 \sigma \int_{0}^{t}\left\|V_{t}\right\|_{0}^{2} \mathrm{~d} t+\delta\|V\|_{0}^{2} \\
& =\|G\|_{0}^{2}+\|\nabla H\|_{0}^{2}+\delta\|H\|_{0}^{2}+2 \int_{0}^{t}\left(F, V_{t}\right) \mathrm{d} t \\
& \quad \leq\|G\|_{0}^{2}+\|\nabla H\|_{0}^{2}+\delta\|H\|_{0}^{2}+\frac{1}{2 \sigma} \int_{0}^{t}\|F\|_{0}^{2} \mathrm{~d} t+2 \sigma \int_{0}^{t}\left\|V_{t}\right\|_{0}^{2} \mathrm{~d} t .
\end{aligned}
$$

Thereupon, when $F(x, y, t)=H_{0}(x, y)=H_{1}(x, y)=0$, we obtain $\|V\|_{0}=\|\nabla V\|_{0}=0$, which implies $u=0$, that is, the weak formulation (2) has a unique solution $V \in H_{0}^{1}(\Theta)$. Further, from (5) we obtain (3). This completes the proof of Theorem 1.

\subsection{The CNFE method for the 2D uniform transmission line equation}

To solve Problem 1 by the CNFE method, let $\Im_{h}$ be a uniformly regular triangulation on $\bar{\Theta}$ (see, e.g., [16]). The FE subspace is defined as

$$
\mathbb{U}_{h}=\left\{v_{h} \in H_{0}^{1}(\Theta) \cap C(\bar{\Theta}):\left.v_{h}\right|_{K} \in \mathbb{P}_{l}(K), K \in \Im_{h}\right\},
$$

where $\mathbb{P}_{l}(K)$ is formed by $l$ th-degree polynomials on $K \in \Im_{h}$.

For integer $N>0$, let $\Delta t=T / N$, and let $V_{h}^{n}$ be the FE approximations to the solutions $V$ in Problem 2 at $t_{n}=n \Delta t(0 \leq n \leq N), \bar{\partial} V_{h}^{n}=\left(V_{h}^{n+1}-V_{h}^{n-1}\right) /(2 \Delta t), \bar{\partial}^{2} V_{h}^{n}=\left(V_{h}^{n+1}-2 V_{h}^{n}+\right.$ $\left.V_{h}^{n-1}\right) / \Delta t^{2}$, and $f^{n}=g\left(x, y, t_{n}\right)$. Then we can establish the following CNFE model for the 2D uniform transmission line equation.

Problem 2 Find $V_{h}^{n} \in \mathbb{U}_{h}$ such that

$$
\left\{\begin{array}{l}
\left(V_{h}^{n+1}-2 V_{h}^{n}+V_{h}^{n-1}, \vartheta_{h}\right)+\frac{\Delta t^{2}}{2}\left(\nabla V_{h}^{n+1}+\nabla V_{h}^{n-1}, \nabla \vartheta_{h}\right) \\
\quad+\frac{\sigma \Delta t}{2}\left(V_{h}^{n+1}-V_{h}^{n-1}, \vartheta_{h}\right)+\frac{\delta \Delta t^{2}}{2}\left(V_{h}^{n+1}+V_{h}^{n-1}, \vartheta_{h}\right) \\
=\Delta t^{2}\left(F\left(t_{h}\right), \vartheta_{h}\right), \quad \vartheta_{h} \in U_{h}, 1 \leq n \leq N-1, \\
V_{h}^{0}(x, y)=R_{h} G(x, y), \quad V_{h}^{1}(x, y)=V_{h}^{0}+2 \Delta t R_{h} H(x, y), \quad(x, y) \in \Theta,
\end{array}\right.
$$

where $F\left(t_{n}\right)=F\left(x, y, t_{n}\right)$, and $P_{h}: \mathbb{U} \rightarrow \mathbb{U}_{h}$ is the Ritz projection (see [16]).

When $g \in H^{l+1}(\Theta) \cap H_{0}^{1}(\Theta)$, the Ritz projection has the following boundedness and error estimates (see, e.g., [16]):

$$
\left\|P_{h} g\right\|_{0} \leq C\|g\|_{0} \quad \text { and } \quad\left\|g-P_{h} g\right\|_{0} \leq C h^{l+1}\|g\|_{l+1}
$$

where $\|\cdot\|_{0}$ and $\|\cdot\|_{l}$ represent, respectively, the norms in $L^{2}(\Theta)$ and $H^{l}(\Theta)$, and $C>0$ stands for a generic constant independent of $\Delta t$ and $h$, different at different places.

Moreover, when $g \in H_{0}^{1}(\Omega)$, it satisfies the Poincaré inequality

$$
C_{p}\|g\|_{1} \leq|g|_{1} \leq\|g\|_{1}
$$

for some $C_{p}>0$.

For Problem 2, we have the existence, uniqueness, and stability of CNFE solutions. 
Theorem 2 If $F \in L^{2}\left(0, T ; L^{2}(\Theta)\right), H_{0} \in H^{1}(\Theta)$, and $H_{1} \in H^{1}(\Theta)$, then Problem 2 has a unique sequence of solutions $V_{h}^{n} \in \mathbb{U}_{h}(n=1,2, \ldots, K)$ satisfying the following unconditionally stability:

$$
\begin{aligned}
\left\|V_{h}^{n}\right\|_{1} \leq & \left(\frac{8+C_{p}^{2}+\delta}{C_{p}^{2} \min \{1, \delta\}}\right)^{1 / 2}\left(\left\|\nabla H_{0}\right\|_{0}+\left\|\nabla H_{1}\right\|_{0}\right) \\
& +\left(\frac{\Delta t}{\sigma \min \{1, \delta\}} \sum_{j=1}^{n}\left\|F\left(t_{j}\right)\right\|_{0}^{2}\right)^{1 / 2} .
\end{aligned}
$$

Proof Since scheme (7) is a linear equation system with respect to $V_{h}^{n+1}$, to prove that Problem 2 has a unique sequence of solutions, it suffices to check that it has merely zero solutions as $H_{0}(x, y)=H_{1}(x, y)=F(x, y, t)=0$.

Choosing $\vartheta_{h}=V_{h}^{n+1}-V_{h}^{n-1}$ in the first equation in (7) and applying the Hölder and Cauchy inequalities, we get

$$
\begin{aligned}
\left\|V_{h}^{n+1}-V_{h}^{n}\right\|_{0}^{2}-\left\|V_{h}^{n}-V_{h}^{n-1}\right\|_{0}^{2}+\frac{\Delta t^{2}}{2}\left(\left\|\nabla V_{h}^{n+1}\right\|_{0}^{2}-\left\|\nabla V_{h}^{n-1}\right\|_{0}^{2}\right) \\
\quad+\frac{\sigma \Delta t}{2}\left\|V_{h}^{n+1}-V_{h}^{n-1}\right\|_{0}^{2}+\frac{\delta \Delta t^{2}}{2}\left(\left\|V_{h}^{n+1}\right\|_{0}^{2}-\left\|V_{h}^{n-1}\right\|_{0}^{2}\right) \\
=\Delta t^{2}\left(F\left(t_{n}\right), V_{h}^{n+1}-V_{h}^{n-1}\right)_{0} \\
\leq \frac{\Delta t^{3}}{2 \sigma}\left\|F\left(t_{n}\right)\right\|_{0}^{2}+\frac{\sigma \Delta t}{2}\left\|V_{h}^{n+1}-V_{h}^{n-1}\right\|_{0}^{2} .
\end{aligned}
$$

Furthermore, we get

$$
\begin{gathered}
\left\|V_{h}^{n+1}-V_{h}^{n}\right\|_{0}^{2}-\left\|V_{h}^{n}-V_{h}^{n-1}\right\|_{0}^{2}+\frac{\Delta t^{2}}{2}\left(\left\|\nabla V_{h}^{n+1}\right\|_{0}^{2}-\left\|\nabla V_{h}^{n-1}\right\|_{0}^{2}\right) \\
+\frac{\delta \Delta t^{2}}{2}\left(\left\|V_{h}^{n+1}\right\|_{0}^{2}-\left\|V_{h}^{n-1}\right\|_{0}^{2}\right) \leq \frac{\Delta t^{3}}{2 \sigma}\left\|F\left(t_{n}\right)\right\|_{0}^{2} .
\end{gathered}
$$

Summing (11) from 1 to $n$, by the second formula in (7) and the Poincaré inequality we get

$$
\begin{aligned}
\left\|V_{h}^{n+1}-V_{h}^{n}\right\|_{0}^{2}+\frac{\Delta t^{2}}{2}\left(\left\|\nabla V_{h}^{n+1}\right\|_{0}^{2}+\left\|\nabla V_{h}^{n}\right\|_{0}^{2}\right)+\frac{\delta \Delta t^{2}}{2}\left(\left\|V_{h}^{n+1}\right\|_{0}^{2}+\left\|V_{h}^{n}\right\|_{0}^{2}\right) \\
\leq\left\|V_{h}^{1}-V_{h}^{0}\right\|_{0}^{2}+\frac{\Delta t^{2}}{2}\left(\left\|\nabla V_{h}^{1}\right\|_{0}^{2}+\left\|\nabla V_{h}^{0}\right\|_{0}^{2}\right) \\
\quad+\frac{\delta \Delta t^{2}}{2}\left(\left\|V_{h}^{1}\right\|_{0}^{2}+\left\|V_{h}^{0}\right\|_{0}^{2}\right)+\frac{\Delta t^{3}}{2 \sigma} \sum_{j=1}^{n}\left\|F\left(t_{j}\right)\right\|_{0}^{2} \\
\leq \frac{4 \Delta t^{2}}{C_{p}^{2}}\left\|\nabla R_{h} H_{0}\right\|_{0}^{2}+\frac{\Delta t^{2}}{2}\left(\left\|\nabla R_{h} H_{0}\right\|_{0}^{2}+\left\|\nabla R_{h} H_{1}\right\|_{0}^{2}\right) \\
\quad+\frac{\delta \Delta t^{2}}{2 C_{P}^{2}}\left(\left\|\nabla R_{h} H_{0}\right\|_{0}^{2}+\left\|\nabla R_{h} H_{1}\right\|_{0}^{2}\right)+\frac{\Delta t^{3}}{2 \sigma} \sum_{j=1}^{n}\left\|F\left(t_{j}\right)\right\|_{0}^{2} \\
\leq\left(\frac{4 \Delta t^{2}}{C_{p}^{2}}+\frac{\Delta t^{2}}{2}+\frac{\delta \Delta t^{2}}{2 C_{P}^{2}}\right)\left(\|\nabla H\|_{0}^{2}+\|\nabla G\|_{0}^{2}\right)+\frac{\Delta t^{3}}{2 \sigma} \sum_{j=1}^{n}\left\|F\left(t_{j}\right)\right\|_{0}^{2}
\end{aligned}
$$


for $n=1,2, \ldots, N-1$. Thereupon, by (12) we have $\left\|\nabla V_{h}^{n}\right\|=\left\|V_{h}^{n}\right\|=0(n=1, \ldots, N)$ as $H_{0}(x, y)=H_{1}(x, y)=F(x, y, t)=0$, which shows that $V_{h}^{n}=0(n=1,2, \ldots, N)$. Hence Problem 2 has a unique set of solutions. By (12) we directly get (9), which finishes the proof of Theorem 2.

The set of solutions for Problem 2 has the following error estimates.

Theorem 3 Under the hypotheses of Theorem 2, we have the following error estimates between the weak solution to Problem 1 and the CNFE solutions to Problem 2:

$$
\left\|V\left(t_{n}\right)-V_{h}^{n}\right\|_{1} \leq C\left(\Delta t^{2}+h^{l}\right), \quad 1 \leq n \leq N
$$

Proof As $V_{t}$ and $V_{t t}$ are, respectively, approximated by $\left(V^{n+1}-V^{n-1}\right) / 2 \Delta t$ and $\left(V^{n+1}-\right.$ $\left.2 V^{n}+V^{n-1}\right) / \Delta t^{2}$, we get the following time semidiscrete scheme for Problem 1 :

$$
\left\{\begin{array}{c}
\left(V^{n+1}-2 V^{n}+V^{n-1}, \vartheta\right)+\frac{\Delta t^{2}}{2}\left(\nabla V^{n+1}+\nabla V^{n-1}, \nabla \vartheta\right) \\
+\frac{\sigma \Delta t}{2}\left(V^{n+1}-V^{n-1}, \vartheta\right)+\frac{\delta \Delta t^{2}}{2}\left(V^{n+1}+V^{n-1}, \vartheta\right) \\
=\Delta t^{2}\left(F\left(t_{n}\right), \vartheta\right), \quad v \in U, 1 \leq n \leq K-1, \\
V^{0}(x, y)=H_{0}(x, y), \quad V^{1}(x, y)=H_{1}(x, y), \quad(x, y) \in \Theta .
\end{array}\right.
$$

Let $E_{1}^{n}=V\left(t_{n}\right)-V^{n}, E_{2}^{n}=V^{n}-R_{h} V^{n}$, and $e_{3}^{n}=R_{h} V^{n}-V_{h}^{n}$.

(1) Estimate for $E_{1}^{n}$.

Using Taylor's formula to (2) at $t=t_{n}$, subtracting (14), and choosing $\vartheta=E_{1}^{n+1}-E_{1}^{n-1}$, by Green's formula and the Hölder and Cauchy inequalities we get

$$
\begin{aligned}
& \left\|E_{1}^{n+1}-E_{1}^{n}\right\|_{0}^{2}-\left\|E_{1}^{n}-E_{1}^{n-1}\right\|_{0}^{2}+\frac{\Delta t^{2}}{2}\left(\left\|\nabla E_{1}^{n+1}\right\|_{0}^{2}-\left\|\nabla E_{1}^{n-1}\right\|_{0}^{2}\right) \\
& +\sigma \Delta t\left\|E_{1}^{n+1}-E_{1}^{n-1}\right\|_{0}^{2}+\delta \Delta t^{2}\left(\left\|E_{1}^{n+1}\right\|_{0}^{2}-\left\|E_{1}^{n-1}\right\|_{0}^{2}\right) \\
& =\frac{\Delta t^{4}}{12}\left(V_{t t t t}\left(\xi_{1}^{n}\right), E_{1}^{n+1}-E_{1}^{n-1}\right)-\frac{\Delta t^{4}}{2}\left(\Delta V_{t t}\left(\xi_{2}^{n}\right), E_{1}^{n+1}-E_{1}^{n-1}\right) \\
& \quad+\frac{\sigma \Delta t^{4}}{6}\left(V_{t t t}\left(\xi_{3}^{n}\right), E_{1}^{n+1}-E_{1}^{n}\right)+\frac{\delta \Delta t^{4}}{2}\left(V_{t t}\left(\xi_{2}^{n}\right), E_{1}^{n+1}-E_{1}^{n-1}\right) \\
& \leq \sigma \Delta t\left\|E_{1}^{n+1}-E_{1}^{n-1}\right\|_{0}^{2}+\frac{\Delta t^{7}}{144 \sigma}\left\|V_{t t t t}\left(\xi_{1}^{n}\right)\right\|_{0}^{2}+\frac{\Delta t^{7}}{4 \sigma}\left\|\Delta V_{t t}\left(\xi_{2}^{n}\right)\right\|_{0}^{2} \\
& +\frac{\sigma \Delta t^{7}}{36}\left\|V_{t t t}\left(\xi_{3}^{n}\right)\right\|_{0}^{2}+\frac{\delta^{2} \Delta t^{7}}{4 \sigma}\left\|V_{t t}\left(\xi_{2}^{n}\right)\right\|_{0}^{2},
\end{aligned}
$$

where $t_{n} \leq \xi_{1}^{n}, \xi_{2}^{n}, \xi_{3}^{n} \leq t_{n+1}$. Since $E_{1}^{1}=E_{1}^{0}=0$, simplifying and summing (15) from 1 to $n$, we get

$$
\begin{aligned}
& 2\left\|E_{1}^{n+1}-E_{1}^{n}\right\|_{0}^{2}+\Delta t^{2}\left(\left\|\nabla E_{1}^{n+1}\right\|_{0}^{2}+\left\|\nabla E_{1}^{n}\right\|_{0}^{2}\right)+2 \delta \Delta t^{2}\left(\left\|E_{1}^{n+1}\right\|_{0}^{2}+\left\|E_{1}^{n}\right\|_{0}^{2}\right) \\
& \quad \leq C^{2}(V) \min \{1,2 \delta\} \Delta t^{6},
\end{aligned}
$$

where $C^{2}(V)=\frac{1}{72 \sigma \min \{1,2 \delta\}}\left[\left\|V_{t t t t}\left(\xi_{1}^{n}\right)\right\|_{0}^{2}+18\left\|\Delta V_{t t}\left(\xi_{2}^{n}\right)\right\|_{0}^{2}+4 \sigma^{2}\left\|V_{t t t}\left(\xi_{3}^{n}\right)\right\|_{0}^{2}+36 \delta^{2}\left\|V_{t t}\left(\xi_{2}^{n}\right)\right\|_{0}^{2}\right]$. Furthermore, we get

$$
\left\|E_{1}^{n}\right\|_{1} \leq C(V) \Delta t^{2}
$$


(2) Estimate for $E_{2}$.

The estimate of $E_{2}$ may be directly obtained from (8) as $V^{n} \in H^{l+1}(\Theta)$ :

$$
\left\|E_{2}^{n}\right\|_{1} \leq C h^{l}, \quad n=1,2, \ldots, N
$$

(3) Estimate for $E_{3}=R_{h} V^{n}-V_{h}^{n}$.

Subtracting Problem 2 from (14) and choosing $\vartheta=\vartheta_{h} \in U_{h}$, we get

$$
\begin{aligned}
& \left(V^{n+1}-V_{h}^{n+1}-2\left(V^{n}-V_{h}^{n}\right)+V^{n-1}-V_{h}^{n-1}, \vartheta_{h}\right) \\
& +\frac{\Delta t^{2}}{2}\left(\nabla\left(V^{n+1}-V_{h}^{n+1}\right)+\nabla\left(V^{n-1}-V_{h}^{n-1}\right), \nabla \vartheta_{h}\right) \\
& \quad+\frac{\sigma \Delta t}{2}\left(V^{n+1}-V_{h}^{n+1}-\left(V^{n-1}-V_{h}^{n-1}\right), \vartheta_{h}\right) \\
& +\frac{\delta \Delta t^{2}}{2}\left(V^{n+1}-V_{h}^{n+1}+V^{n-1}-V_{h}^{n-1}, \vartheta_{h}\right)=0, \quad \vartheta_{h} \in U_{h} .
\end{aligned}
$$

Using the property of $R_{h}$, (8), (19), Taylor's formula, and the Hölder and Cauchy inequalities, we get

$$
\begin{aligned}
\| E_{3}^{n+1}- & E_{3}^{n}\left\|_{0}^{2}-\right\| E_{3}^{n}-E_{3}^{n-1} \|_{0}^{2}+\frac{\Delta t^{2}}{2}\left(\left\|\nabla E_{3}^{n+1}\right\|_{0}^{2}-\left\|\nabla E_{3}^{n-1}\right\|_{0}^{2}\right) \\
& +\frac{\sigma \Delta t}{2}\left\|E_{3}^{n+1}-E_{3}^{n-1}\right\|_{0}^{2}+\delta \Delta t^{2}\left(\left\|E_{3}^{n+1}\right\|_{0}^{2}-\left\|E_{3}^{n-1}\right\|_{0}^{2}\right) \\
= & \left.V^{n+1}-2 V^{n}+V^{n-1}-\left(V_{h}^{n+1}-2 V_{h}^{n}+V_{h}^{n-1}\right), E_{3}^{n+1}-E_{3}^{n-1}\right) \\
& +\left(R_{h} V^{n+1}-V^{n+1}-2\left(R_{h} V^{n}-V^{n}\right)+\left(R_{h} V^{n-1}-V^{n-1}\right), E_{3}^{n+1}-E_{3}^{n-1}\right) \\
& +\frac{\Delta t^{2}}{2}\left(\nabla\left(V^{n+1}-V_{h}^{n+1}\right)+\nabla\left(V^{n-1}-V_{h}^{n-1}\right), \nabla\left(E_{3}^{n+1}-E_{3}^{n-1}\right)\right) \\
& +\frac{\Delta t^{2}}{2}\left(\nabla\left(R_{h} V^{n+1}-V^{n+1}\right)+\nabla\left(R_{h} V^{n-1}-V^{n-1}\right), \nabla\left(E_{3}^{n+1}-E_{3}^{n-1}\right)\right) \\
& +\frac{\delta \Delta t^{2}}{2}\left(V^{n+1}-V_{h}^{n+1}+V^{n-1}-V_{h}^{n-1}, E_{3}^{n+1}-E_{3}^{n-1}\right) \\
& +\frac{\delta \Delta t^{2}}{2}\left(R_{h} V^{n+1}-V^{n+1}+R_{h} V^{n-1}-V^{n-1}, E_{3}^{n+1}-E_{3}^{n-1}\right) \\
= & \left.R_{h} V^{n+1}-V^{n+1}-2\left(R_{h} V^{n}-V^{n}\right)+\left(R_{h} V^{n-1}-V^{n-1}\right), E_{3}^{n+1}-E_{3}^{n-1}\right) \\
& +\frac{\delta \Delta t^{2}}{2}\left(R_{h} V^{n+1}-V^{n+1}+R_{h} V^{n-1}-V^{n-1}, E_{3}^{n+1}-E_{3}^{n-1}\right) \\
\leq & \frac{\Delta t}{2}\left\|E_{3}^{n+1}-E_{3}^{n-1}\right\|_{0}^{2}+C \Delta t^{3} h^{2(l+1)}, \quad n=0,1, \ldots, N-1 .
\end{aligned}
$$

Noting that $E_{3}^{1}=E_{3}^{0}=0$ and summing (20) from 1 to $n$, we get

$$
\begin{aligned}
& \left\|E_{3}^{n+1}-E_{3}^{n}\right\|_{0}^{2}+\frac{\Delta t^{2}}{2}\left(\left\|\nabla E_{3}^{n+1}\right\|_{0}^{2}+\left\|\nabla E_{3}^{n}\right\|_{0}^{2}\right)+\delta \Delta t^{2}\left(\left\|E_{3}^{n+1}\right\|_{0}^{2}+\left\|E_{3}^{n}\right\|_{0}^{2}\right) \\
& \leq C \Delta t^{2} h^{2(l+1)}, \quad n=1,2, \ldots, N .
\end{aligned}
$$


Thereupon, we get

$$
\left\|E_{3}^{n}\right\|_{1} \leq C h^{l+1}, \quad n=1,2, \ldots, N
$$

Uniting (17)-(18) and (22), we gain (13), which completes the proof of Theorem 3.

Remark 1 The order of error estimates in Theorem 3 is optimal. Theorems 2 explains that the CNFE method (Problem 2) for the 2D uniform transmission line equation has a unique set of solutions that is unconditionally stable, so that it is unconditionally convergent and continuously depends on the initial value and source term. This shows that Problem 2 is theoretically reliable and effective for settling the 2D uniform transmission line equation.

\section{Numerical tests}

We employ some numerical tests to verify the correctness of the theoretical consequences of the CNFE method (Problem 2) of the 2D uniform transmission line equation, which can settle out the genuine solution, but usually it has no genuine solution.

In the $2 \mathrm{D}$ uniform transmission line equation (1), we choose $\bar{\Theta}=[-1,1] \times[-1,1]$, $\sigma=2, \delta=1, V_{0}( \pm 1, y, t)=\exp (-t)(1-\cos 2 \pi y)(-1 \leq y \leq 1$ and $t \in[0, T)), V_{0}(x, \pm 1, t)=$ $\exp (-t)(1-\cos 2 \pi x)(-1 \leq x \leq 1$ and $t \in[0, T)), H_{0}(x, y)=1-\cos 2 \pi y \cos 2 \pi x, H_{1}(x, y)=$ $\cos 2 \pi y \cos 2 \pi x-1$, and $F(x, y, t)=8 \pi^{2} \exp (-t) \cos 2 \pi y \cos 2 \pi x$. Thus we can find the following genuine solution for the uniform transmission line equation (1):

$$
V(x, y, t)=\exp (-t)(1-\cos 2 \pi y \cos 2 \pi x), \quad(x, y) \in[-1,1] \times[-1,1], t \in[0, T) .
$$

When choosing time step $\Delta t=0.01$ and adopting the uniformly regular triangulation with $h=0.01$ and the $\mathbb{P}_{2}(K)$ with 2nd-degree polynomials on every triangular element, by Theorem 3 the theoretical errors between the genuine solution and the CNFE solutions $V_{h}^{k}(k=1,2, \ldots, N)$ should be $O\left(10^{-4}\right)$.

Employing the CNFE method (Problem 3), we can compute out the CNFE solutions at $t=0,0.3,0.6,0.9$, depicted in Figs. 1, 2, 3, 4(a), respectively. The genuine solutions at the same time nodes are depicted in Figs. 1, 2, 3, 4(b), respectively. Each pair of plots in Figs. 1, $2,3,4$ is almost the same. The CNFE solutions after $t=0.9$ tend to be stable, which agree with cases of voltage or current in actual uniform transmission line.

Table 1 shows the errors between the genuine solutions and the CNFE solutions and CPU run time for $t=0.3, t=0.6$, and $t=0.9$, respectively, which verifies that the correctness of the theoretical results due to both theoretical and numerical errors reaching

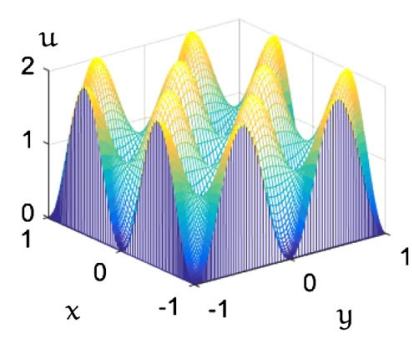

(a)

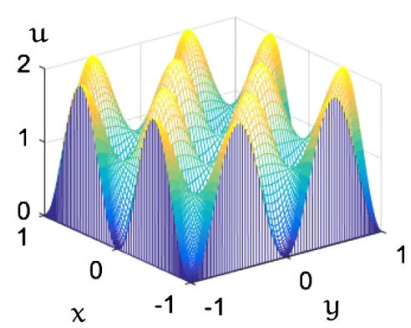

(b)

Figure 1 (a) The genuine solution when $t=0$. (b) The CNFE solution when $t=0$ 


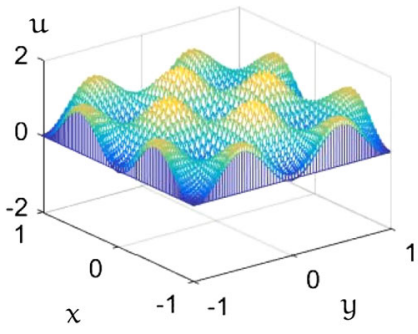

(a)

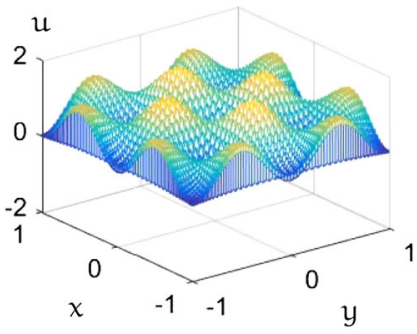

(b)

Figure 2 (a) The genuine solution when $t=0.3$. (b) The CNFE solution when $t=0.3$

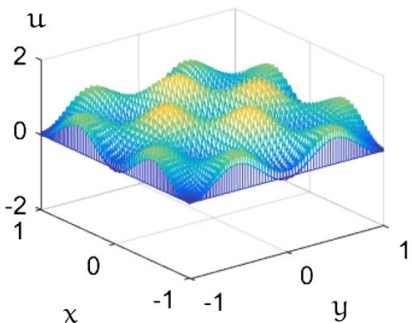

(a)

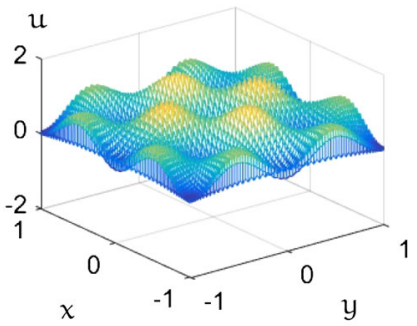

(b)

Figure 3 (a) The genuine solution when $t=0.6$. (b) The CNFE solution when $t=0.6$

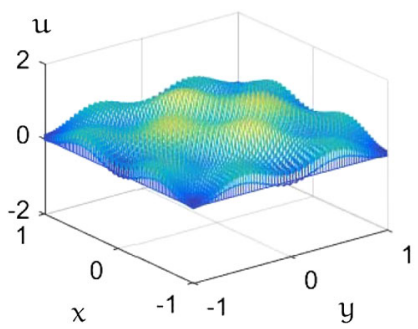

(a)

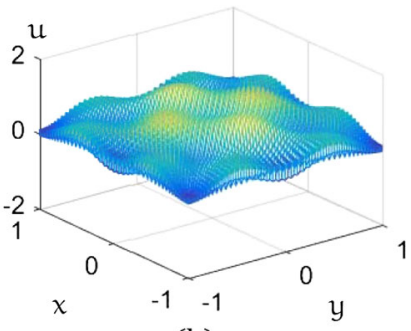

(b)

Figure 4 (a) The genuine solution when $t=0.9$. (b) The CNFE solution when $t=0.9$

$O\left(10^{-4}\right)$, signifies that the CNFE method is effective and reliable for calculating the $2 \mathrm{D}$ uniform transmission line equation. In Table 1, we also give the errors between the genuine and FE solutions and CPU run times when $t=0.3, t=0.6$, and $t=0.9$ using the following FE method:

Problem 3 Find $\tilde{V}_{h}^{n} \in \mathbb{U}_{h}$ such that

$$
\left\{\begin{array}{c}
\left(\tilde{V}_{h}^{n+1}-2 \tilde{V}_{h}^{n}+\tilde{V}_{h}^{n-1}, \vartheta_{h}\right)+\Delta t^{2}\left(\nabla \tilde{V}_{h}^{n}, \nabla \vartheta_{h}\right)+\sigma \Delta t\left(\tilde{V}_{h}^{n}-\tilde{V}_{h}^{n-1}, \vartheta_{h}\right) \\
+\delta \Delta t^{2}\left(\tilde{V}_{h}^{n}, \vartheta_{h}\right)=\Delta t^{2}\left(F\left(t_{n}\right), \vartheta_{h}\right), \quad \vartheta_{h} \in U_{h}, 1 \leq n \leq N-1, \\
\tilde{V}_{h}^{0}(x, y)=R_{h} G(x, y), \quad \tilde{V}_{h}^{1}(x, y)=\tilde{V}_{h}^{0}+\Delta t R_{h} H(x, y), \quad(x, y) \in \Theta,
\end{array}\right.
$$

where $F\left(t_{n}\right)=F\left(x, y, t_{n}\right)$ and $P_{h}: \mathbb{U} \rightarrow \mathbb{U}_{h}$ is the Ritz projection. 
Table 1 The CPU elapsed time and the relative errors of the FE and CNFE solutions

\begin{tabular}{|c|c|c|c|c|c|}
\hline \multirow[t]{2}{*}{$t$} & \multirow[t]{2}{*}{$n$} & \multicolumn{2}{|l|}{ FE method } & \multicolumn{2}{|l|}{ CNFE method } \\
\hline & & $\left\|V\left(t_{n}\right)-\tilde{V}_{h}^{n}\right\|_{1}$ & CPU run time & $\left\|V\left(t_{n}\right)-V_{h}^{n}\right\|_{1}$ & CPU run time \\
\hline 0.3 & 300 & $1.413276 \mathrm{e}-2$ & 1.693 second & $4.051625 \mathrm{e}-5$ & 1.791 second \\
\hline 0.6 & 600 & $3.232292 \mathrm{e}-2$ & 5.897 second & $4.371744 \mathrm{e}-4$ & 6.127 second \\
\hline 0.9 & 900 & $5.482546 \mathrm{e}-2$ & 11.825 second & $4.581633 \mathrm{e}-4$ & 11.982 second \\
\hline
\end{tabular}

It is readily proven that Problem 3 is conditionally stable, so that it is conditionally convergent, and its FE solutions only have the first-order accuracy about time, that is, we have the following error estimates:

$$
\left\|V\left(t_{n}\right)-\tilde{V}_{h}^{n}\right\|_{1} \leq C\left(\Delta t+h^{l}\right), \quad n=1,2, \ldots, N
$$

Therefore, if $\Delta t=0.01$ and $l=2$ (also adopting second-degree elements), then the theoretical errors are of order $O\left(10^{2}\right)$ only, which are consistent with numerical errors; see Table 1. It is further shown that the CNFE method (i.e., Problem 2) has more merits than the FE method (i.e., Problem 3) for settling the 2D uniform transmission line equation.

\section{Conclusions and expectation}

In our study, we have developed the CNFE method for the 2D uniform transmission line equation and analyzed the existence, uniqueness, stability, and errors for the CNFE solutions. We have also adopted some numeric tests to confirm the correctness for the CNFE method. It has been shown, by comparing with the usual FE method, that the CNFE method has more merits. Furthermore, it is revealed that the CNFE method is very effective for settling the 2D uniform transmission line equation.

In spite of the fact that we have only concerned with the CNFE method for the $2 \mathrm{D}$ uniform transmission line equation, the CNFE method may be applied to settle the threedimensional uniform transmission line equations or the uniform transmission line equation with complicated geometrical regions.

Although the CNFE method has many merits, such as unconditional stability, unconditional convergence, and the second-order accuracy in time, it has unknowns (freedom of degrees). When adopting the $\mathbb{P}_{2}(K)$ with 2 nd-degree polynomials on every triangular element in Sect. 3, we may reckon that the CNFE method has about $4 \times 4 \times 10^{4}$ unknowns in the FE method (see [16, Lemma 1.30]). Provided that it is applied for settling big data processing in artificial intelligence and/or computational linguistics, there will be more than tens of millions unknown numbers. Fortunately, a proper orthogonal decomposition (POD) technique may be used to reduce the unknowns in the CNFE method, which can be used to reduce many numerical methods (see, e.g., [17-22]). Our future work is reducing the number of unknowns in the CNFE method by the POD technique.

Acknowledgements

The authors are thankful to the honorable Editors for their valuable suggestions and hard work.

Funding

This research was supported by the National Science Foundation of China grant 11671106. 


\section{Competing interests}

The authors declare that they have no competing interests.

\section{Authors' contributions}

All authors contributed equally and significantly in writing this article. All authors wrote, read, and approved the final manuscript.

\section{Author details}

${ }^{1}$ School of Foreign Studies, University of Science and Technology Beijing, Beijing, China. ${ }^{2}$ School of Mathematics and Physics, North China Electric Power University, Beijing, China.

\section{Publisher's Note}

Springer Nature remains neutral with regard to jurisdictional claims in published maps and institutional affiliations.

Received: 16 March 2020 Accepted: 3 April 2020 Published online: 16 April 2020

\section{References}

1. Hesameddini, E., Asadolahifard, E.: A new spectral Galerkin method for solving the two dimensional hyperbolic telegraph equation. Comput. Math. Appl. 72, 1926-1942 (2016)

2. Mittal, R.C., Bhatia, R.: A collocation method for numerical solution of hyperbolic telegraph equation with Neumann boundary conditions. Int. J. Comput. Math. 2014, Article ID 526814 (2014)

3. Luo, Z.D., Jiang, W.R.: The Crank-Nicolson finite spectral element method and numerical simulations for $2 D$ non-stationary Navier-Stokes equations. Math. Methods Appl. Sci. 43, 2276-2288 (2020)

4. Luo, Z.D., Teng, F., Xia, H.: A reduced-order extrapolated Crank-Nicolson finite spectral element method based on POD for the 2D non-stationary Boussinesq equations. J. Math. Anal. Appl. 71(1-2), 564-583 (2019)

5. Yang, J., Luo, Z.D.: Proper orthogonal decomposition reduced-order extrapolation continuous space-time finite element method for the two-dimensional unsteady Stokes equation. J. Math. Anal. Appl. 475, 123-138 (2019)

6. Teng, F., Luo, Z.D.: A reduced-order extrapolation technique for solution coefficient vectors in the mixed finite element method for the 2D nonlinear Rosenau equation. J. Math. Anal. Appl. 485, Article ID 123761 (2020)

7. Zhou, Y.J, Luo, Z.D.: A Crank-Nicolson collocation spectral method for the two-dimensional telegraph equations. J. Inequal. Appl. 2018, Article ID 137 (2018)

8. He, S., Li, H.: Time discontinuous space-time finite element method for telegraph equations. Appl. Math. J. Chin. Univ. Ser. A 27(4), 425-438 (2012)

9. Mohanty, R.K.: New unconditionally stable difference schemes for the solution of multi-dimensional telegraphic equations. Int. J. Comput. Math. 86(12), 2061-2071 (2009)

10. Hashemi, M.S., Baleanu, D.: Numerical approximation of higher-order time-fractional telegraph equation by using a combination of a geometric approach and method of line. J. Comput. Phys. 316, 10-20 (2016)

11. Biazar, J., Eslami, M.: A new method for solving the hyperbolic telegraph equation. Comput. Math. Model. 23(4), 519-527 (2012)

12. Ma, W.T., Zhang, B.W., Ma, H.L.: A meshless collocation approach with barycentric rational interpolation for two-dimensional hyperbolic telegraph equation. Appl. Math. Comput. 279, 236-248 (2016)

13. Elgindy, K.T.: Higher-order numerical solution of second-order one-dimensional hyperbolic telegraph equation using a shifted Gegenbauer pseudospectral method. Numer. Methods Partial Differ. Equ. 32(1), 307-349 (2016)

14. Wang, H., He, Q.F., Luo, Z.D.: A reduced order extrapolating technique of solution coefficient vectors to collocation spectral method for telegraph equation. Adv. Differ. Equ. 2020, Article ID 61 (2020)

15. Adams, R.A.: Sobolev Spaces. Academic Press, New York (1975)

16. Luo, Z.D.: The Foundations and Applications of Mixed Finite Element Methods. Chinese Science Press, Beijing (2006) (in Chinese)

17. Teng, F., Luo, Z.D., Yang, J.: A reduced order extrapolated natural boundary element method based on POD for the 2D hyperbolic equation in unbounded domain. Math. Methods Appl. Sci. 42, 4273-4291 (2019)

18. Teng, F., Luo, Z.D., Yang, J.: A reduced order extrapolated NBE format based on POD for the 2D parabolic equation in unbounded domain. Comput. Appl. Math. 38(3), Article ID 102 (2019)

19. Luo, Z.D., Jin, S.J.: A reduced-order extrapolated Crank-Nicolson collocation spectral method based on POD for the 2D viscoelastic wave equations. Numer. Methods Partial Differ. Equ. 36, 49-65 (2020)

20. Luo, Z.D., Wang, H.: A highly efficient reduced-order extrapolated finite difference algorithm for time-space tempered fractional diffusion-wave equation. Appl. Math. Lett. 102, Article ID 106090 (2020)

21. Jin, S.J., Luo, Z.D.: A reduced-order extrapolating collocation spectral method based on POD for the 2D Sobolev equations. Bound. Value Probl. 2019, Article ID 63 (2019)

22. Luo, Z.D., Jiang, W.R.: A reduced-order extrapolated finite spectral element method for the 2D non-stationary Navier-Stokes equations about vorticity-stream functions. Appl. Numer. Math. 147, 161-173 (2020) 